\title{
Error Bounds for Nondifferentiable Convex Inequalities under a Strong Slater Constraint Qualification
}

\author{
O. L. Mangasarian* \\ Mathematical Programming Technical Report 96-04 \\ July 1996 - Revised March 1997
}

\begin{abstract}
A global error bound is given on the distance between an arbitrary point in the $n$-dimensional real space $R^{n}$ and its projection on a nonempty convex set determined by $m$ convex, possibly nondifferentiable, inequalities. The bound is in terms of a natural residual that measures the violations of the inequalities multiplied by a new simple condition constant that embodies a single strong Slater constraint qualification (CQ) which implies the ordinary Slater CQ. A very simple bound on the distance to the projection relative to the distance to a point satisfying the ordinary Slater CQ is given first and then used to derive the principal global error bound.
\end{abstract}

Key Words. Convex inequalities, error bounds, Strong Slater constraint qualification.

Abbreviated Title. Error bounds under a strong Slater CQ

\section{Introduction}

We consider the nonempty feasible region

$$
S:=\{x \mid g(x) \leq 0\}
$$

where $g: R^{n} \longrightarrow R^{m}$ is a convex function on the $n$-dimensional real space $R^{n}$. We are interested in bounding the distance between an arbitrary point $x$ in $R^{n}$ and its projection $p(x)$ on the set $S$ in terms of a natural residual $\left\|g(x)_{+}\right\|$, where $\left(g(x)_{+}\right)_{i}:=\max \left\{0, g_{i}(x)\right\}, i=1, \ldots, m$, and $\|\cdot\|$ denotes an arbitrary norm on $R^{m}$. In [10] the author imposed differentiability on $g$, the Slater CQ as well as an asymptotic CQ in order to obtain such an error bound. Auslender and Crouzeix [2] removed the differentiability assumption on $g$ and extended the asymptotic CQ to nondifferentiable convex $g$. Luo and Luo [8] established an error bound for convex quadratic $g$ under the Slater CQ alone. Wang and Pang [12] showed the necessity of the Slater CQ for an error bound to hold for quadratic $g$. Li [7] showed that for convex quadratic $g$, a global error bound holds if and only if Abadie's CQ [1], which implies the Slater CQ, holds. Klatte [4] gave an error bound under the Slater CQ and a "bounded excess" condition. Deng [3] established error bounds for convex inequalities under a Slater condition on recession functions associated with (1). Very recently Lewis and Pang [6] have made a comprehensive study of error bounds for convex inequalities and have

\footnotetext{
*Computer Sciences Department, University of Wisconsin, 1210 West Dayton Street, Madison, WI 53706, email: olvi@cs.wisc.edu This material is based on research supported by National Science Foundation Grant CCR-9322479.
} 
given a number of equivalent conditions for an error bound to hold. Thus so far it appears that for a general convex $g$, an asymptotic or some other $\mathrm{CQ}$ which is complex to state and difficult to verify, is needed in addition to the simple Slater CQ $(\exists \hat{x}: g(\hat{x})<0)$, in order to obtain a global error bound.

In this work, first a very simple global error bound (Theorem 2.2) is obtained on the distance between an arbitrary point $x$ in $R^{n}$ and its projection $p(x)$ on the feasible region $S$ relative to the distance between $x$ and a point $\hat{x}$ in $S$ that satisfies a Slater CQ. This error bound depends, as was the case in [10], on bounding Lagrange multipliers of the problem of projecting $x$ on $S$, and contains no unknown condition constant but merely the natural residual and a measure of satisfaction of the ordinary Slater CQ $\left(\min _{1 \leq i \leq m}-g_{i}(\hat{x})\right)$. By using this bound on the relative error, we next derive our principal result (Theorem 3.4) which gives an error bound on the distance between $x$ and its projection $p(x)$ on $S$. This bound is in terms of the natural residual $\left\|g(x)_{+}\right\|_{\infty}$ and a condition constant that depends on a strong Slater CQ (Definition 3.2) which implies the ordinary Slater $\mathrm{CQ}$, and is characterized by an sup-inf operation (Definition 3.2). An intuitive interpretation of the strong Slater CQ is given by Proposition 3.5 where it is shown that each boundary point of $S$ must be approachable by a sequence of interior points such that directions between the interior points and the boundary point make a uniformly acute angle with the negative gradient of an active constraint at the boundary point.

A word about our notation now. The symbol ":=" denotes definition. Vectors are column vectors unless transposed by a '. For a vector $x$ in the $n$-dimensional real space $R^{n}, x_{+}$will denote the vector in $R^{n}$ with components $\left(x_{+}\right)_{i}:=\max \left\{x_{i}, 0\right\}, i=1, \ldots, n$, while $\|x\|,\|x\|_{1}$ and $\|x\|_{\infty}$, will denote respectively an arbitrary norm, the 1 -norm and the $\infty$-norm of $x$. The notation arg $\min _{x \in X} f(x)$ will denote the set of minimizers of $f(x)$ on the set $X$. For $f: R^{n} \longrightarrow R$ which is differentiable at $x$, the notation $\nabla f(x)$ will represent the $1 \times n$ gradient vector.

\section{A Relative Error Bound under the Ordinary Slater CQ}

In this section we use the ordinary Slater $\mathrm{CQ}$ to bound a relative error. The result depends on bounding Lagrange multipliers associated with the projection problem as follows.

Lemma 2.1 Let $g: R^{n} \longrightarrow R^{m}$ be convex on $R^{n}$ and let $g(\hat{x})<0$ for some $\hat{x} \in R^{n}$. For each $x$ in $R^{n}$ and for an arbitrary norm $\|\cdot\|$ on $R^{n}$, there exist a projection $p(x)$ of $x$ on $S$, that is

$$
p(x) \in \arg \min _{p}\{\|p-x\| \mid g(p) \leq 0\},
$$

and an optimal Lagrange multiplier $w(x) \geq 0$ in $R^{m}$, such that $(p(x), w(x))$ satisfy the KarushKuhn-Tucker saddle-point conditions:

$$
L(p(x), w, x) \leq L(p(x), w(x), x) \leq L(p, w(x), x), \forall p \in R^{n}, \forall w \in R^{m}, w \geq 0,
$$

where $L(p, w, x)$ is the Lagrangian associated with the projection problem (2) for a fixed $x$, that is

$$
L(p, w, x):=\|p-x\|+w^{\prime} g(p) .
$$

Furthermore,

$$
\|w(x)\|_{1} \leq \frac{\|x-\hat{x}\|-\|x-p(x)\|}{\min _{1 \leq i \leq m}-g_{i}(\hat{x})} .
$$


Proof The satisfaction of the KKT saddle-point conditions (3), as well as

$$
w(x)^{\prime} g(p(x))=0,
$$

follow from the convexity of $\|p-x\|$ in $p$ for a fixed $x$ and the Slater CQ [9, Theorem 5.4.7]. We now establish (5). From (3) and (6) we have, for a fixed $x$, that

$$
\|p(x)-x\|=\|p(x)-x\|+w(x)^{\prime} g(p(x)) \leq\|\hat{x}-x\|+w(x)^{\prime} g(\hat{x}) .
$$

Hence

$$
\|w(x)\|_{1} \cdot \min _{1 \leq i \leq m}-g_{i}(\hat{x}) \leq-w(x)^{\prime} g(\hat{x}) \leq\|\hat{x}-x\|-\|p(x)-x\|,
$$

from which (5) follows.

With the bound (5) on the optimal multiplier $w(x)$, we can derive our relative error bound.

Theorem 2.2 Relative Error Bound Let $g: R^{n} \longrightarrow R^{m}$ be convex on $R^{n}$ and let $g(\hat{x})<0$ for some $\hat{x} \in R^{n}$. For each $x$ in $R^{n}, x \neq \hat{x}$, the distance to its projection $p(x)$ on $S$ is relatively bounded as follows:

$$
\frac{\|x-p(x)\|}{\|x-\hat{x}\|} \leq \frac{\left\|g(x)_{+}\right\|_{\infty}}{\left\|g(x)_{+}\right\|_{\infty}+\min _{1 \leq i \leq m}-g_{i}(\hat{x})} .
$$

Proof From the saddle-point conditions (3) we have

$$
\|p(x)-x\| \leq\|x-x\|+w(x)^{\prime} g(x) \leq\|w(x)\|_{1} \cdot\left\|g(x)_{+}\right\|_{\infty} \cdot
$$

By using the bound (5) from Lemma 2.1 we obtain

$$
\|p(x)-x\| \leq \frac{\|x-\hat{x}\|-\|x-p(x)\|}{\min _{1 \leq i \leq m}-g_{i}(\hat{x})}\left\|g(x)_{+}\right\|_{\infty},
$$

which gives the desired bound (8).

With the help of the relative error bound (8) and an appropriately strengthened Slater CQ, we can derive our global error bound.

\section{A Global Error Bound under a Strong Slater CQ}

We begin this section by defining the strong Slater CQ. For that purpose we define the interior and boundary of the feasible region $S$ in terms of our convex and hence continuous function $g$ on $R^{n}$.

\section{Definition 3.1}

$$
\begin{aligned}
\text { int } S & :=\{x \mid g(x)<0\} \\
\partial S & :=\left\{x \mid g(x) \leq 0, g_{\ell}(x)=0 \text { for some } \ell, 1 \leq \ell \leq m\right\}
\end{aligned}
$$

These definitions of interior and boundary points coincide with the corresponding conventional definitions of convex analysis if a Slater CQ is assumed.

Definition 3.2 Strong Slater CQ There exists a positive number $\gamma$ such that

$$
\sup _{p \in \partial S} \inf _{\hat{x} \in \text { int } S} \frac{\|\hat{x}-p\|}{\min _{1 \leq i \leq m}-g_{i}(\hat{x})} \leq \gamma<\infty .
$$


An immediate consequence of the strong Slater CQ is the following.

Lemma 3.3 Equivalent Strong Slater CQ For $\hat{\gamma}>\gamma$, the strong Slater CQ (9) implies that

$$
\forall p \in \partial S \exists \hat{x}(p) \in \operatorname{int} S: \frac{\|\hat{x}(p)-p\|}{\min _{1 \leq i \leq m}-g_{i}(\hat{x}(p))} \leq \hat{\gamma}<\infty .
$$

Conversely, (10) implies the strong Slater $C Q(9)$ with $\gamma=\hat{\gamma}$.

Proof From (9) we have that

$$
\forall p \in \partial S: \inf _{\hat{x} \in \text { int } S} \frac{\|\hat{x}-p\|}{\min _{1 \leq i \leq m}-g_{i}(\hat{x})} \leq \gamma<\infty
$$

From the definition of infimum we have from (11) that for $\hat{\gamma}>\gamma$ :

$$
\forall p \in \partial S \exists \hat{x}(p) \in \operatorname{int} S: \frac{\|\hat{x}(p)-p\|}{\min _{1 \leq i \leq m}-g_{i}(\hat{x}(p))}<\inf _{\hat{x} \in \operatorname{int} S} \frac{\|\hat{x}-p\|}{\min _{1 \leq i \leq m}-g_{i}(\hat{x})}+(\hat{\gamma}-\gamma) .
$$

Combining (11) and (12) gives (10).

To establish the converse we have from (10) that

$$
\forall p \in \partial S: \inf _{\hat{x} \in \text { int } S} \frac{\|\hat{x}-p\|}{\min _{1 \leq i \leq m}-g_{i}(\hat{x})} \leq \hat{\gamma}<\infty
$$

Taking the supremum over $p \in \partial S$ gives (9) with $\gamma=\hat{\gamma}$.

We are prepared now to derive our principal global error bound.

Theorem 3.4 Global Error Bound Let $g: R^{n} \longrightarrow R^{m}$ be convex on $R^{n}$, let the strong Slater $C Q$ (9) hold and let $\hat{\gamma}>\gamma$. Then for each $x$ in $R^{n}$, the distance to its projection $p(x)$ on $S$ is bounded as follows:

$$
\|x-p(x)\| \leq \hat{\gamma}\left\|g(x)_{+}\right\|_{\infty},
$$

where $\|\cdot\|$ is an arbitrary norm on $R^{n}, p(x)$ is defined by (2), and hence both $\gamma$ and $p(x)$ are norm-dependent.

Proof For $x \in S, p(x)=x$ and (13) holds for arbitrary $\hat{\gamma}$. Suppose now $x$ is not in $S$ and let $p(x)$ be its projection on $S$. By Lemma 3.3 there exists $\hat{x}(p(x)) \in$ int $S$ such that the inequality of (10) holds with $p=p(x)$, that is

$$
\frac{\|\hat{x}(p(x))-p(x)\|}{\min _{1 \leq i \leq m}-g_{i}(\hat{x}(p(x)))} \leq \hat{\gamma}<\infty .
$$

With $\hat{x}=\hat{x}(p(x))$ in $(8)$, we conclude by Theorem 2.2 that,

$$
\begin{aligned}
\|x-p(x)\| & \leq \frac{\|x-\hat{x}(p(x))\|-\|x-p(x)\|}{\min _{1 \leq i \leq m}-g_{i}(\hat{x}(p(x)))}\left\|g(x)_{+}\right\|_{\infty} \\
& \leq \frac{\|x-p(x)\|+\|p(x)-\hat{x}(p(x))\|-\|x-p(x)\|}{\min _{1 \leq i \leq m}-g_{i}(\hat{x}(p(x)))}\left\|g(x)_{+}\right\|_{\infty}
\end{aligned}
$$

(By triangle inequality)

$\leq \hat{\gamma}\left\|g(x)_{+}\right\|_{\infty}(\mathrm{By}(14))$. 
We give now a sufficient condition for satisfying the strong Slater CQ. This condition, which sheds some intuitive light on the constraint qualification, requires that each boundary point $p$ of $S$, with some active constraint $g_{\ell}(p)=0$, be approachable by a sequence of interior points $\left\{\hat{x}^{j}\right\}$ such that the directions $\left\{\hat{x}^{j}-p\right\}$ make a uniformly acute angle with $-\nabla g_{\ell}(p)$ and such that $g_{\ell}\left(\hat{x}^{j}\right) \leq g_{i}\left(\hat{x}^{j}\right), i=1, \ldots, m$.

Proposition 3.5 Sufficient Condition for Strong Slater CQ Let $g: R^{n} \longrightarrow R^{m}$ be differentiable and convex on $R^{n}$. The strong Slater $C Q(9)$ is satisfied with $\gamma=\hat{\gamma}$ if for each $p$ on the boundary $\partial S$ of $S$, there exists an active constraint index $\ell \in\{1, \ldots, m\}$ and a sequence of interior points $\left\{\hat{x}^{j}\right\} \subset$ int $S$, converging to $p$, such that for some $\hat{\gamma}>0$ and some $\epsilon>0$ :

$$
g_{\ell}(p)=0, \ell \in \arg \min _{1 \leq i \leq m}-g_{i}\left(\hat{x}^{j}\right),-\nabla g_{\ell}(p) \frac{\hat{x}^{j}-p}{\left\|\hat{x}^{j}-p\right\|} \geq \frac{1+\epsilon}{\hat{\gamma}}, j=1,2, \ldots
$$

Proof By the differentiability of $g_{\ell}$ at $p \in \partial S$, we have that

$$
g_{\ell}(p)-g_{\ell}\left(\hat{x}^{j}\right)=-\nabla g_{\ell}(p)\left(\hat{x}^{j}-p\right)-\alpha\left(p, \hat{x}^{j}-p\right)\left\|\hat{x}^{j}-p\right\|,
$$

where $\lim _{\hat{x}^{j}-p \rightarrow 0} \alpha\left(p, \hat{x}^{j}-p\right)=0$. Hence

$$
\begin{aligned}
-g_{\ell}\left(\hat{x}^{j}\right)=g_{\ell}(p)-g_{\ell}\left(\hat{x}^{j}\right) & =\left\|\hat{x}^{j}-p\right\|\left(-\nabla g_{\ell}(p) \frac{\left(\hat{x}^{j}-p\right)}{\left\|\hat{x}^{j}-p\right\|}-\alpha\left(p, \hat{x}^{j}-p\right)\right), j=1,2, \ldots \\
& \geq\left\|\hat{x}^{j}-p\right\|\left(\frac{1+\epsilon}{\hat{\gamma}}-\alpha\left(p, \hat{x}^{j}-p\right)\right), j=1,2, \ldots(\text { By }(15)) \\
& \geq \frac{\left\|\hat{x}^{j}-p\right\|}{\hat{\gamma}}, \text { for } j \geq \bar{j} \text { for some } \bar{j} .
\end{aligned}
$$

Hence (10) is satisfied by $\left(p, \hat{x}^{\bar{j}}\right)$, and by Lemma 3.3, the strong Slater CQ (9) holds with $\gamma=\hat{\gamma}$. $\square$

We conclude this section by giving a simple example demonstrating the use of the sufficient condition (15) and showing how the constant $\hat{\gamma}$ bounds $\sup _{x \notin S} \frac{\|x-p(x)\|}{\left\|g(x)_{+}\right\|}$from above, as it must by $(13)$.

\section{Example 3.6}

$$
S:=\left\{x \mid x \in R^{2}, e^{-x_{1}}-x_{2} \leq 0\right\}
$$

For this example

$$
g(x)=e^{-x_{1}}-x_{2}, \nabla g(x)=\left[\begin{array}{ll}
-e^{-x_{1}} & -1
\end{array}\right]
$$

If we take a point $p$ on the boundary $\partial S$ of $S$, that is $e^{-p_{1}}-p_{2}=0$, and $\left\{\hat{x}^{j}\right\} \in$ int $S$ on the normal to $\partial S$ at $p$, and such that:

$$
\hat{x}^{j}=\left[\begin{array}{l}
p_{1}+\delta_{j} e^{-p_{1}} \\
e^{-p_{1}}+\delta_{j}
\end{array}\right], 0<\left\{\delta_{j}\right\} \longrightarrow 0,
$$

then condition (15) is satisfied as follows

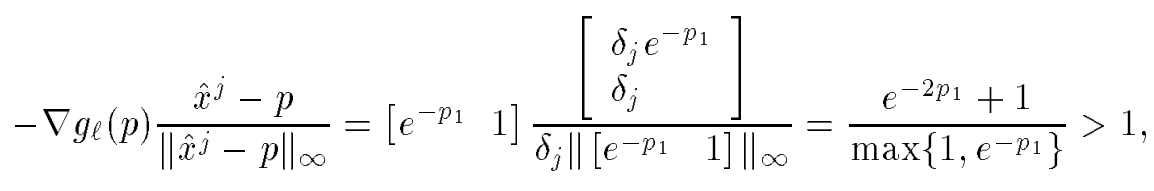


where the infimum 1 is approached as $p_{1} \longrightarrow \infty$. It follows by (15) that $\hat{\gamma}=1+\epsilon$. To see that $\hat{\gamma}$ bounds $\sup _{x \notin S} \frac{\|x-p(x)\|}{\|g(x)+\|}$, we compute $\sup _{x \notin S} \frac{\|x-p(x)\|_{\infty}}{\|g(x)+\|_{\infty}}$, where $p(x)$ is the projection of $x$ on $S$ using the infinity norm. For any $x \notin S$, the projection $p(x)$ is the intersection with $S$ of the smallest square centered at $x$ with sides parallel to the coordinate axes. Denoting the components of $p(x)$ by $\left(p_{1}, p_{2}\right)$ and making the sides of the square equal gives for $x_{1}<p_{1}$ :

$$
e^{-p_{1}}-x_{2}=p_{1}-x_{1} \quad \text { or equivalently } x_{2}=x_{1}-p_{1}+e^{-p_{1}}
$$

Hence

$$
\frac{\|x-p(x)\|_{\infty}}{\|g(x)+\|_{\infty}}=\frac{p_{1}-x_{1}}{e^{-x_{1}}-x_{2}}=\frac{p_{1}-x_{1}}{e^{-x_{1}}-x_{1}+p_{1}-e^{-p_{1}}} \leq 1
$$

where the supremum of 1 is approached as $\left(p_{1}-x_{1}\right) \longrightarrow 0$ and $x_{1} \longrightarrow \infty$. Thus $\sup _{x \notin S} \frac{\|x-p(x)\|_{\infty}}{\|g(x)+\|_{\infty}}=$ $1<1+\epsilon=\hat{\gamma}$.

Any example violating the Slater CQ also violates the Strong Slater CQ. However it is not easy to construct an example violating the Strong Slater CQ but not the Slater CQ. In fact this is essentially one of the open challenges also posed by Klatte and Li [5].

\section{Conclusion}

By bounding Lagrange multipliers of the problem of projecting an arbitrary point on a given convex set by means of a Slater or strong Slater constraint qualification, global error bounds on the relative and absolute distances to the convex set were obtained in terms of a natural residual multiplied by a condition constant depending on the point satisfying the constraint qualification.

Acknowledgments I am indebted to my colleague Jong-Shi Pang for making an earlier version of [6] available to me after finishing this paper. Pang [11,6] has also shown that the strong Slater CQ (9) implies a more general strong Slater CQ [6] based on convex analysis and which also implies an error bound. The condition constant $\gamma$ appearing explicitly in our strong Slater CQ (9) can also be obtained from the strong Slater CQ of [6], by an inf-sup operation as suggested there. The author is also indebted to a referee for pointing out that after the completion of this paper, Klatte and Li [5] showed that under the Slater CQ, the Strong Slater CQ 3.2 is equivalent to the Asymptotic CQ of Auslender and Crouzeix [2]. In addition, Klatte and Li [5] showed the necessity of the Sufficient Condition (15), appropriately extended using subgradients to nondifferentiable convex $g(x)$, for the Strong Slater CQ (9) [5, Theorem 6, ACQ4

\section{References}

[1] J. Abadie. On the Kuhn-Tucker theorem. In J. Abadie, editor, Nonlinear Programming, pages 19-36. North-Holland, Amsterdam, 1967.

[2] A. A. Auslender and J.-P. Crouzeix. Global regularity theorems. Mathematics of Operations Research, 13:243-253, 1988.

[3] S. Deng. Computable error bounds for convex inequality systems in reflexive Banach spaces. SIAM Journal on Optimization, 7:274-279, 1997.

[4] D. Klatte. Hoffman's error bound for systems of convex inequalities. Working paper, Institut Operations Research, Universität Zürich, CH-8044 Zürich, 1996. 
[5] D. Klatte and W. Li. Asymptotic constraint qualifications and global error bounds for convex inequalities. Working paper, Institut Operations Research, Universität Zürich, CH-8044 Zïrich, 1996.

[6] A. S. Lewis and J.-S. Pang. Error bounds for convex inequality systems. Working paper, Department of Mathematical Sciences, The Johns Hopkins University, Baltimore, MD 21218, June 1996.

[7] W. Li. Abadie's constraint qualification, metric regularity, and error bounds for differentiable convex inequalities. SIAM Journal on Optimization, 1996. To appear.

[8] X.-D. Luo and Z.-Q. Luo. Extension of hoffman's error bound to polynomial systems. SIAM Journal on Optimization, 4:383-392, 1994.

[9] O. L. Mangasarian. Nonlinear Programming. McGraw-Hill, New York, 1969. Reprint: SIAM Classic in Applied Mathematics 10, 1994, Philadelphia.

[10] O. L. Mangasarian. A condition number for differentiable convex inequalities. Mathematics of Operations Research, 1:175-179, 1985.

[11] J.-S. Pang. Private communication, July 1996.

[12] T. Wang and J.-S. Pang. Global error bounds for convex quadratic inequality systems. Optimization, 31:1-12, 1994. 